\title{
Effect of Quantum Confinement on the Dielectric Function of PbSe
}

\author{
Z. Hens* and D. Vanmaekelbergh \\ Debye Institute, Utrecht University, P.O. Box 80000, 3508 TA Utrecht, The Netherlands
}

E. S. Kooij and H. Wormeester

$\mathrm{MESA}^{+}$Research Institute, University of Twente, P.O. Box 217, 7500 AE Enschede, The Netherlands

G. Allan and C. Delerue

IEMN (CNRS, UMR 8520), Département ISEN, 41 Boulevard Vauban, 59046 Lille CEDEX, France

(Received 27 June 2003; published 16 January 2004)

\begin{abstract}
Monolayers of lead selenide nanocrystals of a few nanometers in height have been made by electrodeposition on a $\mathrm{Au}(111)$ substrate. These layers show a thickness-dependent dielectric function, which was determined using spectroscopic ellipsometry. The experimental results are compared with electronic structure calculations of the imaginary part of the dielectric function of PbSe nanocrystals. We demonstrate that the size-dependent variation of the dielectric function is affected by quantum confinement at well-identifiable points in the Brillouin zone, different from the position of the band-gap transition.
\end{abstract}

DOI: 10.1103/PhysRevLett.92.026808

Semiconductor nanocrystals with dimensions below $10 \mathrm{~nm}$ possess optical properties that depend strongly on the size and shape of the nanocrystals. This is due to confinement of the exciton wave function in the limited space of the nanocrystal [1]. In many cases, the sizedependent optical properties can be understood by considering the effects of quantum confinement on the band-gap transition, i.e., the electronic transition with lowest energy, and a few other closely related transitions [2]. However, quantum confinement will affect all electronic transitions throughout the entire Brillouin zone and, thus, decide the dielectric function of a semiconductor nanocrystal in a broad energy range. Despite the scientific and technological importance, only a few studies have been published in which the dielectric function of semiconductors nanocrystals is considered $[3,4]$.

Here, we present a study on the effects of quantum confinement on the dielectric function of $\mathrm{PbSe}$ nanocrystals in the energy range $1.5-4 \mathrm{eV}$. Bulk PbSe is a low band-gap semiconductor, with a band-gap transition of $0.26 \mathrm{eV}$ at room temperature (at the $L$ point of the Brillouin zone) and several other critical points (Van Hove singularities) at higher energy (at the $L$ point and along the $\Delta$ and $\Sigma$ directions). PbSe nanocrystals form an ideal case for this study: They can be electrodeposited on a gold substrate with good control over their size and shape [5], and the effects of quantum confinement are expected to be strong due to the low effective masses of the electrons and holes [6]. We studied the dielectric function $\epsilon(\omega)$ of PbSe nanocrystals deposited on gold by spectroscopic ellipsometry. The dielectric function of the PbSe nanocrystals can be described by a sum of two Lorentz oscillators, as reported before for bulk PbSe [7]. This shape of the dielectric function is determined by an increase of the oscillator strength and the joint density of
PACS numbers: 73.22.Dj, 73.63.-b

states near the Van Hove singularities along the directions $\Delta$ and $\Sigma$ in the Brillouin zone [8]. We found that the resonance energies of these oscillators increase strongly relative to the bulk values when the nanocrystal height is less than $5 \mathrm{~nm}$. This blueshift is accompanied by a change of the oscillator width and the oscillator strength. These results are compared with a tight-binding calculation of the dielectric function of an infinite $\mathrm{PbSe}$ sheet with varying height. We demonstrate that this profound change of $\epsilon(\omega)$ can be understood only by considering the effects of quantum confinement on the energy bands throughout the entire Brillouin zone. In this way, the present study reveals that quantum confinement can have an important influence on the dielectric function of semiconductor nanostructures.

We deposited PbSe nanocrystal layers from an aqueous solution of lead acetate $(2.5 \mathrm{mM})$ and selenous acid $(1.25 \mathrm{mM})$ [9] on evaporated gold substrates at a potential of $-0.5 \mathrm{~V}$ vs saturated calomel. The growth of crystalline $\mathrm{PbSe}$ was confirmed by $\mathrm{x}$-ray diffraction analysis at layers from 3 to $100 \mathrm{~nm}$ in thickness and by transmission electron microscopy at the very thin layers ( 2 to $5 \mathrm{~nm}$ ). The topography of the nanocrystals was studied with room-temperature scanning tunneling microscopy. The layers are composed of densely packed nanocrystals with edges ranging from 15 to $25 \mathrm{~nm}$, as shown by Fig. 1(a). These edges did not vary considerably with the nanocrystal height for heights exceeding 2-3 $\mathrm{nm}$. Therefore, the charge consumed in the electrodeposition is a good estimate of the height of the PbSe nanocrystals. For deposits with a height below 2-3 nm, nanocrystals with edges varying from 2.5 to $25 \mathrm{~nm}$ can be found [5]. Resonant tunneling spectroscopy of the energy level structure of the nanocrystals shows that nanocrystals with edges of $15-25 \mathrm{~nm}$ and a height of a few $\mathrm{nm}$ have 

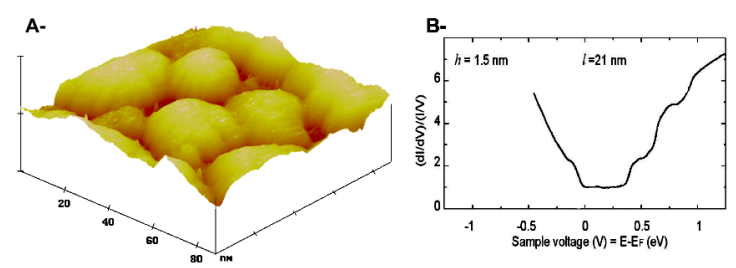

FIG. 1 (color online). (a) STM image of PbSe nanocrystals on a flame-annealed gold surface. Nanocrystal height estimated from charge deposited: $4 \mathrm{~nm}$. (b) Tunneling spectrum obtained at a $\mathrm{PbSe}$ nanocrystal with a height of $1.5 \mathrm{~nm}$ and edges of $21 \mathrm{~nm}$. For more details, see Ref. [5].

a density of states (DOS) close to that of a 2D semiconductor [see Fig. 1(b)] [5]. When the edges are reduced, the DOS of the nanocrystals gradually changes to that of a OD semiconductor. We conclude that our deposition procedure yields a monolayer of 2D nanocrystals with tunable height, keeping in mind that deposits below 2-3 nm in height contain more confined nanocrystals as well.

The color of a monolayer of PbSe nanocrystals varies strongly with the height of the nanocrystals (Fig. 2). Since the energy gap, as determined by scanning tunneling spectroscopy, is below $0.5-1 \mathrm{eV}$ (near IR) for all nanocrystals [5] on the film, this color variation is not due to confinement effects on the PbSe band-gap transition. We investigated this change of the optical properties by spectroscopic ellipsometry at $\mathrm{PbSe}$ nanocrystals of varying height $(2-15 \mathrm{~nm})$. With this technique, one measures the ratio between the (complex) reflection coefficients for light polarized parallel $\left(r_{p}\right)$ and perpendicular $\left(r_{s}\right)$ to the substrate surface. This ratio is typically written in the form $r_{p} / r_{s}=\tan (\Psi) e^{\mathrm{i} \Delta}$. For a multilayer system, an approximation of an unknown $\epsilon(\omega)$ can be obtained by fitting a model function with adjustable parameters to the ellipsometric data $\tan (\Psi)$ and $\cos (\Delta)$, if the respective dielectric functions of the other layers are known.

Figure 2 represents ellipsometric spectra, obtained at bare gold and at the $\mathrm{PbSe} \mid \mathrm{Au}$ system for three different $\mathrm{PbSe}$ nanocrystal heights. As the height of the nanocrystals increases, a systematic change in the spectra occurs. We analyzed the ellipsometric spectra using an air|PbSe|gold three-layer model in the energy range 1.5$3.5 \mathrm{eV}$. The dielectric function of gold was obtained from measurements on a bare gold substrate. The dielectric function of the layer of PbSe nanocrystals is modeled as a sum of two Lorentz oscillators:

$$
\epsilon(E)=\epsilon_{\mathrm{inf}}+\frac{f_{1}}{E_{01}^{2}-E^{2}-\mathrm{i} E \Gamma_{1}}+\frac{f_{2}}{E_{02}^{2}-E^{2}-\mathrm{i} E \Gamma_{2}} .
$$

In this expression, $E_{01}$ and $E_{02}$ are the resonance energies of the oscillators, $f_{1}$ and $f_{2}$ are the oscillator strengths, and $\Gamma_{1}$ and $\Gamma_{2}$ are the oscillator widths. This model was chosen since the description of the dielectric function of

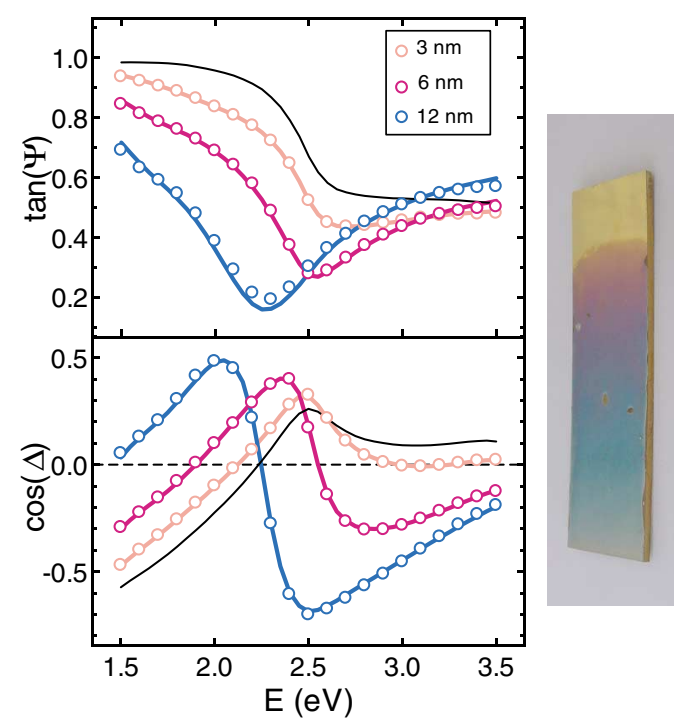

FIG. 2 (color). (left panel) Ellipsometric spectra obtained on bare gold (full line) and on gold samples with a monolayer of $\mathrm{PbSe}$ nanocrystals with thicknesses of 3, 6, and $12 \mathrm{~nm}$, respectively. For clarity, only one in two data points is shown. The full lines represent the results of a fit using a planar threelayer system where the dielectric function of $\mathrm{PbSe}$ is described as a sum of two Lorentz oscillators. (right panel) Image of a flame-annealed gold surface on which a layer of $\mathrm{PbSe}$ nanocrystals was electrodeposited. During electrodeposition, the sample was retracted from the plating solution, leading to an increase of the nanocrystal height from $2 \mathrm{~nm}$ (top) to $20 \mathrm{~nm}$ (bottom). As the thickness increases, the color of the layer changes from pink over purple and blue to gray.

$\mathrm{PbSe}$ in the visible as a sum of two Lorentz oscillators has been reported before for bulk $\mathrm{PbSe}$ [7]. As demonstrated by Fig. 2, this model function fits the measured ellipsometric spectra very well.

The layer thickness as obtained from the data fits is proportional to the charge deposited and corresponds to a deposition efficiency of $75 \%-85 \%$. The proportionality between layer thickness and charge deposited confirms the idea of one dimensional growth of the nanocrystals. Rather than the layer thickness obtained from each individual fit, we used this conversion efficiency — it being an average over various samples - to estimate the nanocrystals height from the charge deposited.

Figure 3 shows the imaginary part $\epsilon_{i m}$ of the dielectric function of the $\mathrm{PbSe}$ layer, as obtained by fitting the ellipsometric data between 1.5 and $3.5 \mathrm{eV}$ for nanocrystals with a height of 1.6 and $8.8 \mathrm{~nm}$, respectively. The contribution of both Lorentz oscillators is indicated and the resonance energies $E_{01}$ and $E_{02}$ are drawn. Since the width of the oscillator is considerable, the resonance energies are slightly shifted relative to the maximum of $\epsilon_{i m}$. The spectrum of $\epsilon_{i m}$ obtained at the $8.8 \mathrm{~nm}$ nanocrystals resembles that of bulk $\mathrm{PbSe}$ [7]. Figure 4 represents the resonance energy of the oscillators as a function of the nanocrystal height. Both figures show a profound change 

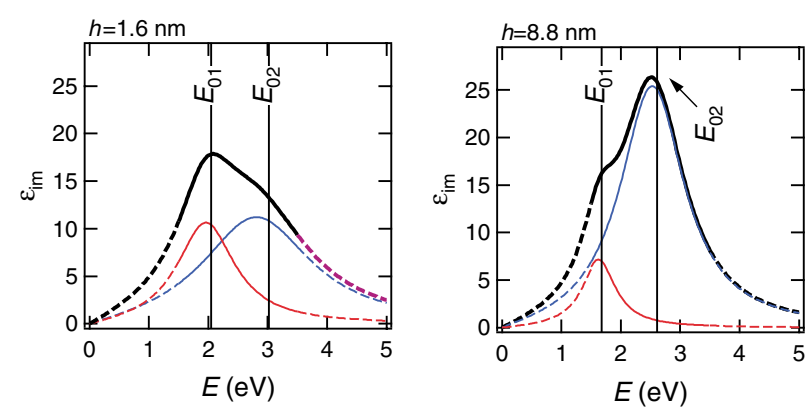

FIG. 3 (color online). Imaginary part of the dielectric function of a layer of PbSe nanocrystals with a height of 1.6 (left) and $8.8 \mathrm{~nm}$ (right). The individual contributions of both Lorentz oscillators are drawn and the resonance energies $E_{01}$ and $E_{02}$ are indicated. In the energy range where the ellipsometric spectra have been fitted, the data are drawn in full.

of the dielectric function on reducing the nanocrystal height. First, the relative contribution of both oscillators to the overall dielectric function strongly changes. Whereas oscillator 2 dominates $\epsilon_{i m}$ for bulk PbSe, both oscillators contribute about equally to $\epsilon_{i m}$ when the nanocrystal height is reduced to $1.6 \mathrm{~nm}$ (Fig. 3). Second, above $5 \mathrm{~nm}$, the resonance energies decrease slowly and tend to the values reported for bulk $\mathrm{PbSe}\left(E_{1}=1.60 \mathrm{eV}\right.$ and $E_{2}=2.73 \mathrm{eV}$, respectively). However, there is a strong increase of the two resonance energies with decreasing height below $5 \mathrm{~nm}$, amounting to a shift of $0.4-0.5 \mathrm{eV}$ at a height of $1.6 \mathrm{~nm}$ (Fig. 4).

To interpret the observed evolution of the dielectric function with varying nanocrystal height, we have calculated the imaginary part $\epsilon_{i m}$ of the dielectric function of $\mathrm{PbSe}$ "sheets" with infinite edges and variable height along the (001) direction. The bulk electronic structure is obtained from a tight-binding approach using an $s p^{3} d^{5} s^{*}$ basis and taking into account a screening surface potential [10]. Our calculations show that the Lorentz

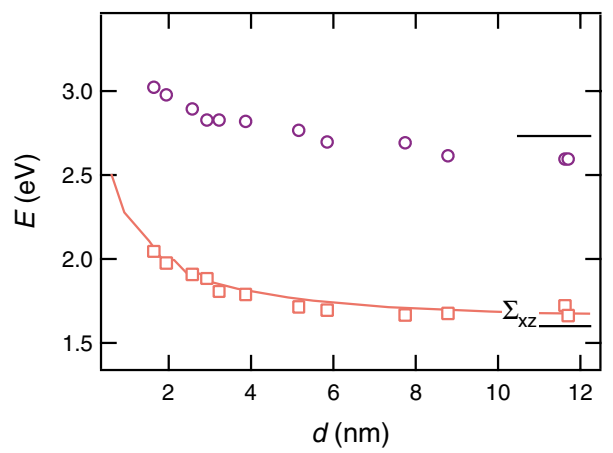

FIG. 4 (color online). Resonance energy of the Lorentz oscillators as a function of the PbSe layer thickness, the squares representing $E_{01}$, the circles $E_{02}$. The full line represents the energy gap at the Van Hove singularity along $\Sigma_{x z}$. The short horizontal lines represent the resonance energies for bulk $\mathrm{PbSe}$ as reported in Ref. [7]. oscillators in the dielectric function are not physically real in the sense that they describe a single electronic transition. Rather, they emerge from an increase of the joint density of states and the oscillator strength of valence-to-conduction band transitions near the energy gap minima (Van Hove singularities) along the $\Sigma$ direction (oscillator 1 at $1.6 \mathrm{eV}$ ) and the $\Delta$ direction (oscillator 2 at $2.7-3 \mathrm{eV}$ ). This means that $\boldsymbol{\epsilon}_{x}$ is determined by states along the directions $\Delta_{x}, \Sigma_{x y}$, and $\Sigma_{x z}$ [14] and the same holds, mutatis mutandum for $\epsilon_{y}$ and $\epsilon_{z}$. In a good approximation, the upper valence-band and the lower conduction-band eigenstates along $\Delta_{x}\left(\Delta_{y}, \Delta_{z}\right)$ are linear chains of $p_{x}\left(p_{y}, p_{z}\right)$ states, whereas the eigenstates along $\Sigma_{x y}\left(\Sigma_{x z}, \Sigma_{y z}\right)$ are linear combinations of $p_{x}$ and $p_{y}\left(p_{x}\right.$ and $p_{z}, p_{y}$ and $p_{z}$ ) states [15]. Consequently, there is no confinement effect to first order on the states along $\Delta_{x}, \Delta_{y}$, and $\Sigma_{x y}$ when the height ( $z$ direction) is reduced. This means that height reduction leads to an anisotropic dielectric function. The dielectric function $\epsilon_{z}$ is affected by confinement effects along $\Delta_{z}, \Sigma_{x z}$, and $\Sigma_{y z}$. Hence, both the shoulder and the peak of $\epsilon_{z}$ will shift. On the other hand, $\epsilon_{x}$ and $\epsilon_{y}$ are affected only by confinement via states along $\Sigma_{x z}$ and $\Sigma_{y z}$, respectively. Hence, only the shoulder at $1.6 \mathrm{eV}$ will vary in this case. This first-order picture is confirmed by the dielectric function as obtained from tight-binding calculations using the full $s p^{3} d^{5} s^{*}$ basis. Figure 5 shows two examples, calculated at sheets with heights of 1.24 and $6.20 \mathrm{~nm}$, respectively. In the latter case, $\epsilon_{i m}$ resembles closely the dielectric function for bulk PbSe [8]. One sees that $\epsilon_{z, i m}$ shows a strong blueshift when the height is reduced. This blueshift is much stronger along $\Delta_{z}$ than along the $\Sigma$ directions. For $\epsilon_{x y, i m}$, a blueshift of the shoulder at $1.6 \mathrm{eV}$ is seen, whereas the main peak only shows a slight redshift.

Figure 6 presents an experimental ellipsometric spectrum obtained at a $1.6 \mathrm{~nm}$ deposit and two (almost coincident) theoretical spectra calculated from the theoretical dielectric function of a $1.55 \mathrm{~nm}$ thick PbSe sheet. One is obtained taking the anisotropy of $\epsilon$ into account, whereas the second assumes a layer with an isotropic dielectric function equal to $\epsilon_{x y}$. First, since the isotropic
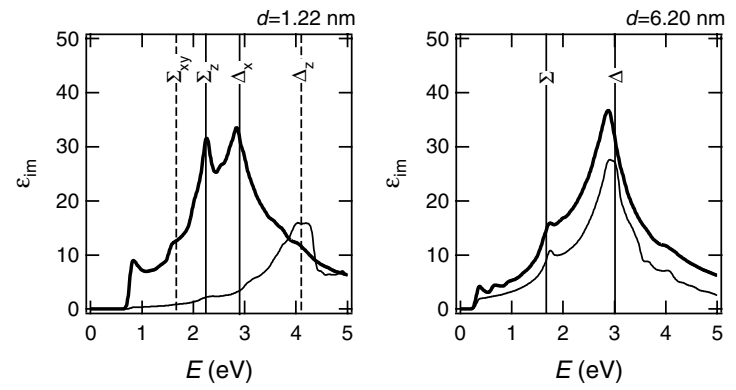

FIG. 5. Calculated spectrum of $\epsilon_{i m}$ for a PbSe sheet with infinite edges and a height of 1.24 and $6.2 \mathrm{~nm}$, respectively, showing $\epsilon_{x y}$ (bold) and $\epsilon_{z}$ (normal). The energy gap minima along the directions $\Delta_{x}, \Delta_{z}, \Sigma_{x y}$, and $\Sigma_{x z}$ are indicated. 


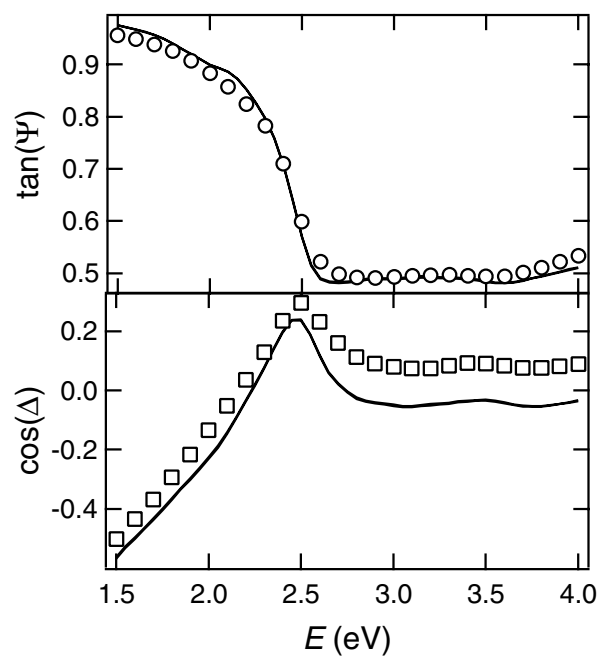

FIG. 6. Comparison of spectroscopic ellipsometry data as measured at a monolayer of PbSe nanocrystals on gold (estimated height: $1.6 \mathrm{~nm}$ ) and as calculated for a PbSe "sheet" with a height of $1.55 \mathrm{~nm}$. The spectra obtained for an isotropic and an anisotropic dielectric function almost coincide.

and anisotropic spectra coincide, Fig. 6 demonstrates that our experimental setup is sensitive to $\epsilon_{x y}$ only. Second, the figure shows that there is a good correspondence between the experimental data and the theoretical calculations. This is confirmed by a comparison of Figs. 3 and 5, which shows that the "experimental" $\epsilon_{i m}$ resembles closely the theoretical dielectric function $\epsilon_{x y}$. Both the imaginary part of the experimental dielectric function (Fig. 3) and the calculated dielectric function (Fig. 5) evolve from a single peak with a low energy shoulder for thick layers $(d>5 \mathrm{~nm})$ to a function with two peaks of equal importance at very thin layers. The fact that the experimental system is a layer of separate nanocrystals rather than an infinite sheet of $\mathrm{PbSe}$ may account for the lower magnitude of the experimental $\epsilon_{i m}$. As demonstrated in Fig. 4, the resonance energy of the first Lorentz oscillator $\left(E_{01}\right)$ follows closely the minimum energy gap along the $\Sigma_{x z}$ and $\Sigma_{y z}$ directions. This is in full accordance with our theoretical results. On the other hand, theory predicts an almost invariant value for $E_{02}$, while our experiments show a blueshift also in this case. However, especially for the deposits below $3 \mathrm{~nm}$ in height, confinement in the $x$ and $y$ direction will complicate the measurements since nanocrystals with edges below $5 \mathrm{~nm}$ will be present. This may explain the observed blueshift of $E_{02}$. The inhomogeneous size distribution of the nanocrystals may account for the broadening of the Lorentz oscillators in the experimental dielectric function (inhomogeneous broadening) as well.
We conclude that this work demonstrates that the dielectric function of low-dimensional semiconductors can be strongly influenced by the effects of quantum confinement. The effects depend in a complex way on the band structure throughout the entire Brillouin zone. In the case of PbSe crystals confined in one direction of space, a strongly anisotropic dielectric function is predicted. Experimentally, the lateral dielectric function $\epsilon_{x y}$ could be determined. These experiments demonstrate that confinement in PbSe nanocrystals leads to a change of the energy and the relative strength of the electronic transitions that determine the dielectric function.

*Electronic address: Zeger.Hens@UGent.be Present address: Laboratorium voor Fysische Chemie, Ghent University, 281-S12, 9000 Gent, Belgium.

[1] L. E. Brus, J. Chem. Phys. 80, 4403 (1984).

[2] D. J. Norris, A. L. Efros, M. Rosen, and M. G. Bawendi, Phys. Rev. B 53, 16347 (1996).

[3] M. Shim and P. Guyot-Sionnest, J. Chem. Phys. 111, 6955 (1999).

[4] L.-W. Wang and A. Zunger, Phys. Rev. B 53, 9579 (1996).

[5] Z. Hens et al. (to be published).

[6] F. Wise, Acc. Chem. Res. 33, 773 (2000).

[7] N. Suzuki, K. Sawai, and S. Adachi, J. Appl. Phys. 77, 1249 (1995).

[8] S. E. Kohn, P.Y. Yu, Y. Petroff, Y. R. Shen, Y. Tsang, and M. L. Cohen, Phys. Rev. B 8, 1477 (1973).

[9] A. N. Molin and A. I. Dikusar, Thin Solid Films 265, 3 (1995).

[10] The tight-binding parameters were fitted on the valenceand conduction-band effective masses [11] and on the $a b$ initio bulk band structure calculated with the abinit code [12] in the local density approximation (LDA) and the Hartwigsen-Goedecker-Hutter pseudopotentials. The bulk band gap underestimated by the LDA approximation has been adjusted to the experimental value with the usual scissor operator. To calculate $\epsilon_{i m}$, we have taken the interatomic matrix elements of the momentum operator equal to those of the effective momentum operator defined in [13]. They are equal to the product of the interatomic tight-binding parameters between two orbitals $\alpha$ and $\beta\left(=s, p, d\right.$, or $\left.s^{*}\right)$ located on nearest neighbor sites $R_{\alpha}$ and $R_{\beta}$ and the factor $e\left(R_{\alpha}-R_{\beta}\right)$.

[11] K. F. Cuff, M. R. Ellet, D. Kuglin, and L. R. Williams, Proceedings of the 7th International Conference on Physics of Semiconductors, Paris, 1964, edited by M. Hulin (Dunod, Paris, 1964), p. 677.

[12] X. Gonze et al., Comput. Mater. Sci. 25, 478 (2002).

[13] M. Graf and P. Vogl, Phys. Rev. B 51, 4940 (1995).

[14] The symbol $\Delta_{x}$ denotes the (100) direction in the Brillouin zone, $\Delta_{z}$ is the (001) direction, etc.

[15] G. Allan, Phys. Rev. B 43, 9594 (1991). 